\title{
LIN28 cooperates with WNT signaling to drive invasive intestinal and colorectal adenocarcinoma in mice and humans
}

\author{
Ho-Chou Tu, ${ }^{1,2,3}$ Sarah Schwitalla, ${ }^{1,2,3}$ Zhirong Qian, ${ }^{4}$ Grace S. LaPier, ${ }^{1,2,3}$ Alena Yermalovich, ${ }^{1,2,3}$ \\ Yuan-Chieh Ku, ${ }^{5}$ Shann-Ching Chen, ${ }^{5}$ Srinivas R. Viswanathan, ${ }^{1,2,3}$ Hao Zhu, ${ }^{6}$ Reiko Nishihara, ${ }^{4}$ \\ Kentaro Inamura, ${ }^{4}$ Sun A. Kim, ${ }^{4}$ Teppei Morikawa, ${ }^{4}$ Kosuke Mima, ${ }^{4}$ Yasutaka Sukawa, ${ }^{4}$ Juhong Yang, ${ }^{4}$ \\ Gavin Meredith, ${ }^{5}$ Charles S. Fuchs, ${ }^{4}$ Shuji Ogino, ${ }^{4,7,8,9}$ and George Q. Daley ${ }^{1,2,3,10}$ \\ ${ }^{1}$ Division of Pediatric Hematology/Oncology, Boston Children's Hospital, Boston, Massachusetts 02115, USA; ${ }^{2}$ Dana Farber \\ Cancer Institute, Boston, Massachusetts 02115, USA; ${ }^{3}$ Harvard Stem Cell Institute, Boston, Massachusetts 02115, USA; \\ ${ }^{4}$ Department of Medical Oncology, Dana Farber Cancer Institute, Boston, Massachusetts 02115, USA; ${ }^{5}$ Thermo Fisher Scientific, \\ Incorporated, South San Francisco, California 94080, USA; ${ }^{6}$ Children's Medical Center Research Institute, University of Texas \\ Southwestern, Dallas, Texas 75390, USA; ${ }^{7}$ Department of Pathology, Brigham and Women's Hospital, Boston, Massachusetts \\ 02115, USA; ${ }^{8}$ Harvard Medical School, Boston, Massachusetts 02115, USA; ${ }^{9}$ Department of Epidemiology, Harvard School of \\ Public Health, Boston, Massachusetts 02115, USA; ${ }^{10}$ Howard Hughes Medical Institute, Boston, Massachusetts 02138, USA
}

Colorectal cancer (CRC) remains a major contributor to cancer-related mortality. LIN28A and LIN28B are highly related RNA-binding protein paralogs that regulate biogenesis of let-7 microRNAs and influence development, metabolism, tissue regeneration, and oncogenesis. Here we demonstrate that overexpression of either LIN28 paralog cooperates with the Wnt pathway to promote invasive intestinal adenocarcinoma in murine models. When LIN28 alone is induced genetically, half of the resulting tumors harbor Ctnnb1 ( $\beta$-catenin) mutation. When overexpressed in $\mathrm{Apc}^{\mathrm{Min} /+}$ mice, LIN28 accelerates tumor formation and enhances proliferation and invasiveness. In conditional genetic models, enforced expression of a LIN28-resistant form of the let-7 microRNA reduces LIN28-induced tumor burden, while silencing of LIN28 expression reduces tumor volume and increases tumor differentiation, indicating that LIN28 contributes to tumor maintenance. We detected aberrant expression of LIN28A and/or LIN28B in $38 \%$ of a large series of human CRC samples $(n=595)$, where LIN28 expression levels were associated with invasive tumor growth. Our late-stage CRC murine models and analysis of primary human tumors demonstrate prominent roles for both LIN28 paralogs in promoting CRC growth and progression and implicate the LIN28/let-7 pathway as a therapeutic target.

[Keywords: LIN28; let-7 miRNA; WNT; oncogene cooperation; invasive adenocarcinoma; colorectal cancer]

Supplemental material is available for this article.

Received December 1, 2014; revised version accepted April 15, 2015.

Colorectal cancer (CRC), one of the most common types of cancer worldwide, is associated with high mortality rates (http://globocan.iarc.fr/Default.aspx). CRC is caused by multiple genetic and epigenetic alterations, but the first major mutational event typically affects the WNT oncogenic pathway, as at least $80 \%$ of sporadic CRCs display loss-of-function mutations of the APC tumor suppressor gene (Fearon and Vogelstein 1990; Kinzler and Vogelstein 1996). However, it is documented that a mutational event in $A P C$ is not sufficient to maintain a pathologic level of WNT activity (Brabletz et al. 2001; Blaker et al. 2003; Fodde and Brabletz 2007). Moreover, perturbations in the RAS, TP53, DNA repair, and other pathways

Corresponding author: george.daley@childrens.harvard.edu Article published online ahead of print. Article and publication date are online at http://www.genesdev.org/cgi/doi/10.1101/gad.256693.114. are often observed to cooperate with the WNT pathway and promote further tumor progression (Fearon 2011). Most of the known drivers in CRC development are essential in normal intestinal homeostasis and thus render them unsuitable as therapeutic targets. Furthermore, the existing mouse models mostly exhibit intestinal adenomas or local adenocarcinomas that seldom progress to highly invasive forms (Janssen et al. 2006; Taketo and Edelmann 2009). Therefore, generating robust late-stage CRC models and identifying novel players contributing to disease progression would provide relevant preclinical

(C) $2015 \mathrm{Tu}$ et al. This article is distributed exclusively by Cold Spring Harbor Laboratory Press for the first six months after the full-issue publication date (see http://genesdev.cshlp.org/site/misc/terms.xhtml). After six months, it is available under a Creative Commons License (Attribution-NonCommercial 4.0 International), as described at http:// creativecommons.org/licenses/by-nc/4.0/. 
platforms and assist the identification of novel drug targets for more effective therapeutics.

LIN28 is an evolutionarily conserved RNA-binding protein with roles in development, tissue regeneration, and tumorigenesis (Viswanathan et al. 2009; Zhu et al. 2011; Shyh-Chang and Daley 2013; Shyh-Chang et al. 2013). Originally described in Caenorhabditis elegans as a heterochronic gene, Lin 28 controls proper developmental timing of the C. elegans larvae (Ambros and Horvitz 1984; Moss et al. 1997). In mammals, LIN28A and its paralog, LIN28B, are expressed in embryonic stem cells (ESCs) and early embryos (Moss and Tang 2003; Yang and Moss 2003). LIN28A and LIN28B maintain stem cell function at least in part by disrupting the terminal processing of the let-7 microRNA (miRNA) family precursors, thus resulting in reduced levels of mature let-7 (Heo et al. 2008; Newman et al. 2008; Viswanathan et al. 2008). Let-7 promotes cell differentiation and maintains tissue homeostasis of various adult cell types as they negatively regulate an array of genes that promote cell cycle entry and self-renewal (Johnson et al. 2005; Lee and Dutta 2007; Mayr et al. 2007; Zhu et al. 2011). Indeed, mature let-7 miRNAs are widely detected among terminally differentiated adult tissues in which LIN28A and LIN28B are mostly absent (Moss and Tang 2003; Yang and Moss 2003; Bussing et al. 2008).

Impaired differentiation is a common feature of advanced human malignancies, many of which display gene expression profiles that overlap with ESCs (Ben-Porath et al. 2008; Kim and Orkin 2011). Previously, our group and others have reported high levels of LIN28A and/or LIN28B in a range of solid and hematological malignancies (Viswanathan et al. 2009; Zhou et al. 2013), especially those with undifferentiated features and poor prognostic outcome. Interestingly, while some cancers such as testicular germ cell tumor and prostate cancer tend to overexpress LIN28A /Gillis et al. 2011; Nadiminty et al. 2012), other cancer types-including Wilms tumor (Urbach et al. 2014), neuroblastoma (Diskin et al. 2012; Molenaar et al. 2012), and hepatocellular carcinoma (Guo et al. 2006; Nguyen et al. 2014)—exclusively overexpress LIN28B. Additionally, some germ cell tumors, breast cancer, and CRC are reported to overexpress both paralogs (West et al. 2009; Piskounova et al. 2011). Given such heterogeneity, it is unclear whether the two paralogs play redundant or independent roles in the tumor development process. Transgenic overexpression of mouse or human forms of LIN28B have produced murine models of T-cell acute lymphoid leukemia (Beachy et al. 2012), neuroblastoma (Molenaar et al. 2012), Wilms tumor (Urbach et al. 2014), hepatocellular carcinoma (Nguyen et al. 2014), and intestinal tumors, but the earlier demonstration of intestinal tumorigenesis did not assess the role of LIN28A and concluded that LIN28B could act as an oncogene in the absence of other canonical genetic alterations (Madison et al. 2013). Typically, cancers require perturbations in more than one oncogenic pathway, and, to date, the interaction between LIN28 and other oncogenic pathways during tumor development has not been rigorously defined.
Because CRC is one of the cancer types reported to overexpress both LIN28 paralogs and is a classic paradigm of multistep oncogenesis, we systematically interrogated the roles of both LIN28A and LIN28B in CRC in both murine models and human samples and explored the potential involvement of cooperating oncogenic pathways. Here we demonstrate important roles of both LIN28 paralogs in tumor initiation and progression.

\section{Results}

Lin28a and Lin28b are expressed in embryonic but not adult intestines

We first explored the expression patterns of Lin28 in normal mouse intestines and found that Lin28a and Lin $28 b$ proteins were expressed at high levels in the early embryonic gut followed by a rapid decline after embryonic day 13.5 (E13.5), which coincides with a critical transition in intestinal epithelial differentiation (Gregorieff and Clevers 2005). The expression of Lin28a and Lin28b was undetectable in the adult small intestine and colon (Fig. 1A,B; Supplemental Fig. 1A). Our conclusion that Lin28b expression is lacking in the adult gut contrasts with a recent study in which Lin28b staining was detected in the adult small intestine and colon by immunohistochemistry (Madison et al. 2013). Using the same Lin28b antibody used in this prior study and examining control tissues from Lin28b knockout animals (Shinoda et al. 2013), we conclude that Lin28b expression is lacking in the adult gut (Supplemental Fig. 1B).

To test whether reactivation of LIN28A is equally as oncogenic as LIN28B and whether there are any critical differences between the transforming properties of the two paralogs, we generated transgenic mice with intestinespecific overexpression of mouse Lin28a or human LIN28B (hereafter referred as iLin28a and iLIN28B or collectively as iLIN28) (Supplemental Fig. 2A,B; Urbach et al. 2014). To model reactivation, we fed doxycycline to adult mice but noted rapid deterioration and death in most of the iLin28a mice (Supplemental Fig. 2C). In contrast, induction of LIN28B in adults was well tolerated. Fortuitously, we were able to maintain iLin28a animals when they were exposed to doxycycline beginning in utero and thereafter. Therefore, the iLin28a animals that we report in this study were induced beginning in utero, while iLIN28B animals were induced as adults (in utero induction of LIN28B produced similar results). These data, alongside the differential effects of Lin28a and LIN28B on intestinal differentiation noted below, suggest distinct roles for the two paralogs in intestinal development and pathology.

LIN28 promotes proliferation and inhibits differentiation of the intestinal epithelium

The intestinal crypts consist of two proliferating populations: the Lgr5 ${ }^{+}$intestinal stem cells (ISCs) at the crypt base and the transit-amplifying (TA) cells, the progenitors located in the middle of the crypts that are the direct 
Tu et al.

A
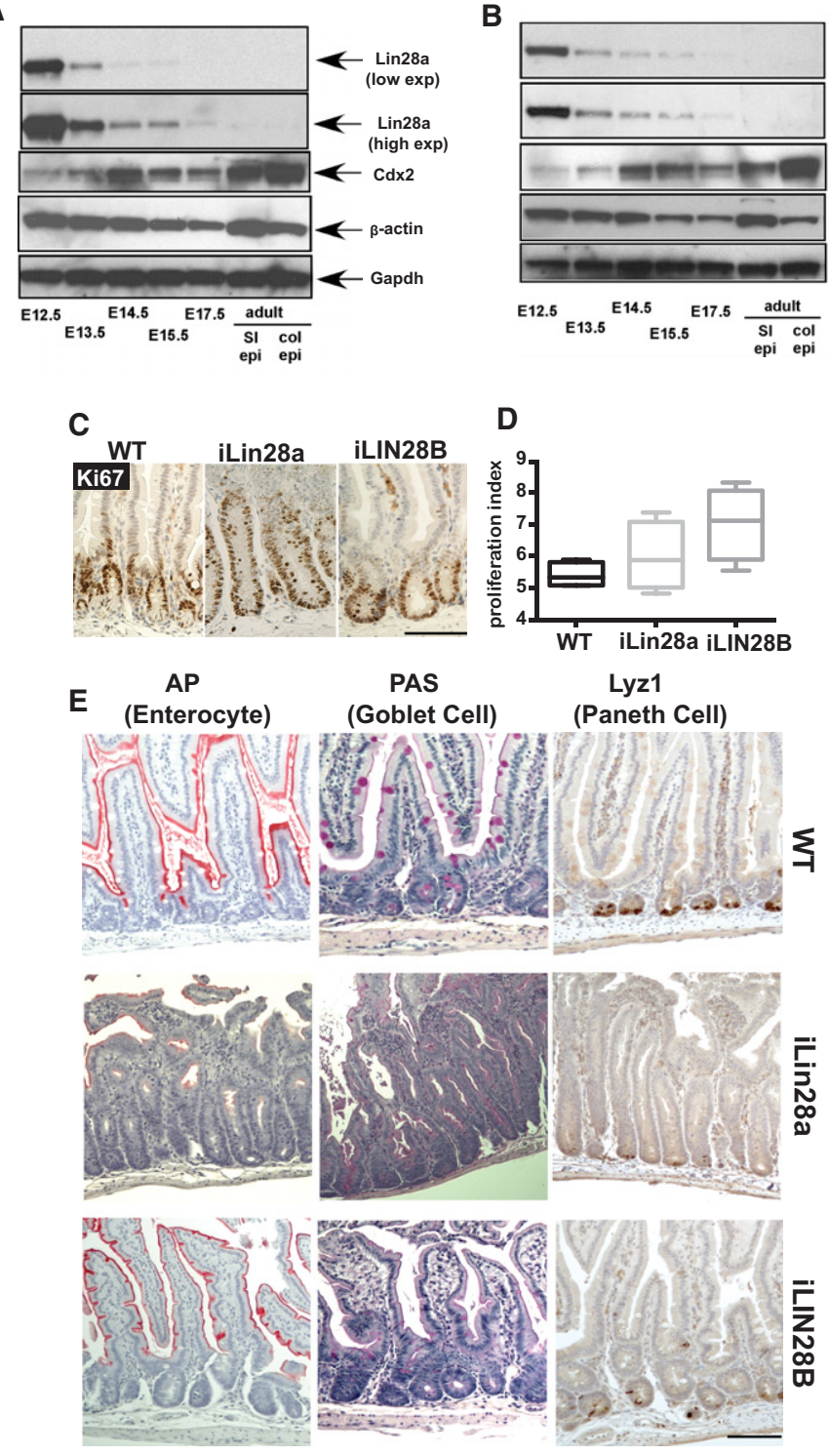

B

D

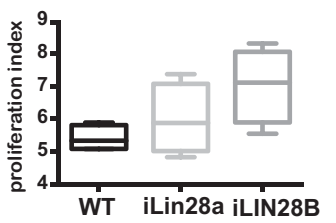

Lyz1

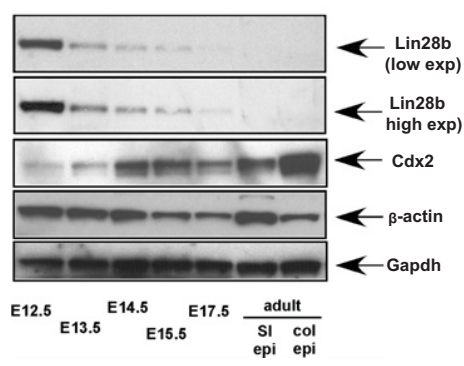

daughters of ISCs. Descendants of the TA progenitors exit the cell cycle and differentiate into specific epithelial lineages, including enterocytes, Paneth cells, goblet cells, neuroendocrine cells, $M$ cells, and tuft cells /Clevers 2013). During differentiation, cells migrate out of the crypts to the villi compartments, except for the Paneth cells, which migrate to the crypt base, where they intercalate with the ISCs and provide a favorable niche that sustains ISC self-renewal (Sato et al. 2011). Strikingly, intestinal induction of LIN28 expression resulted in strong perturbations to the highly ordered intestinal epithelia. Within a few days to a couple of weeks after doxycycline treatment, we observed a significant increase in the proliferating population (Fig. 1C,D; Supplemental Fig. 2D) and loss of certain differentiated cell types, including the Paneth cells and goblet cells. Interestingly, only iLin28a, but not iLIN28B, resulted in loss of the enterocyte lineage even when induced on similar sched-
Figure 1. Ectopic LIN28 expression results in increased proliferation and loss of differentiation in the intestinal epithelium. $(A, B)$ Western blot analysis against Lin28a and Lin28b in lysates collected from dissected embryonic guts at the indicated embryonic days. Adult samples are epithelial cells isolated from the small intestine and colon. (Exp) Exposure time. (C) Representative images of anti-Mki67 immunohistochemistry staining of wild-type (WT), iLin28a, and iLIN28B small intestinal crypts. Bar, $100 \mu \mathrm{m}$. $(D)$ iLin28a and iLIN28B crypts showing increased proliferation index (calculated by the number of Mki67 $7^{+}$ cells/the number of Mki67- cells). (E) Representative images of alkaline phosphatase (AP), periodic acidSchiff (PAS) staining, and anti-Lyz (lysozyme) immunostaining of wild-type, iLin28a, and iLIN28B intestinal mucosa. Bar, $100 \mu \mathrm{m}$. ules (Fig. 1E). Altogether, these phenotypes indicate that acute reactivation of LIN28 in the adult intestinal epithelia provokes increased self-renewal and loss of differentiation potential of the intestinal stem/progenitor cells.

\section{LIN28 activation cooperates with Ctnnb1 mutation to drive invasive adenocarcinoma}

Following 6 mo of doxycycline exposure, almost all iLin 28 transgenic animals developed tumors in the small intestines but, interestingly, never in the colon. LIN28-induced intestinal tumors varied in multiplicity, with a median of three tumors per animal at 6-9 mo after induction (Fig. 2A; Supplemental Fig. 3A). Tumors appeared either dome-like or with a flat morphology and ranged from adenoma to high-grade adenocarcinoma that invaded deep into the muscularis (Fig. 2B). Further supporting the 
A

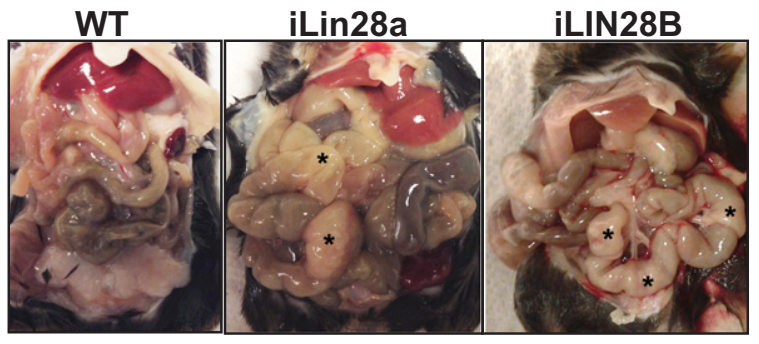

C
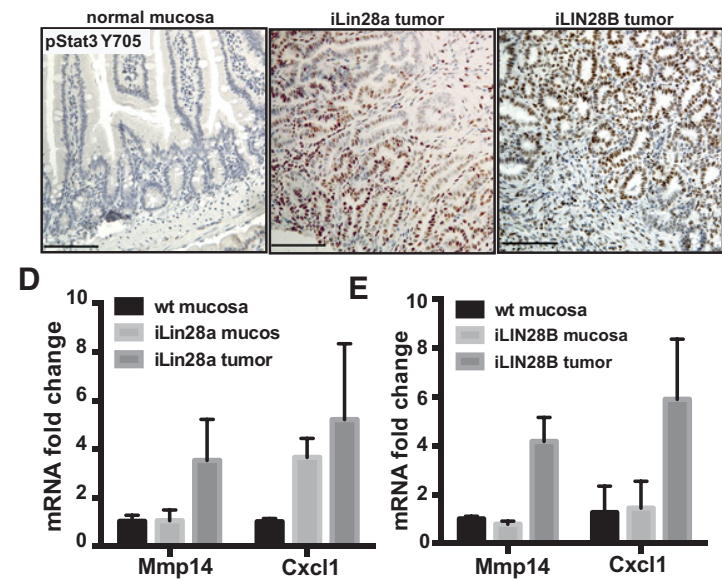

$J$

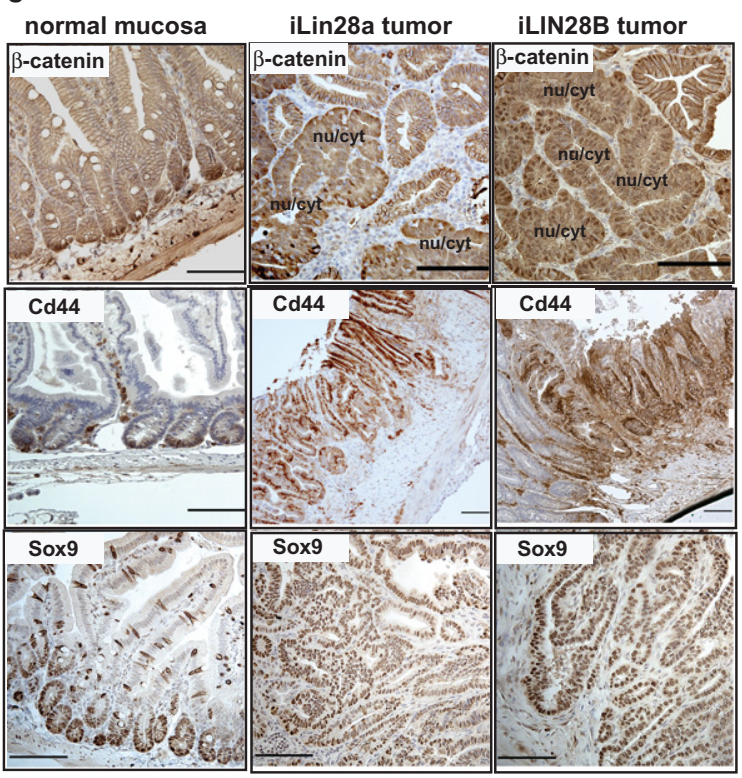

M
B
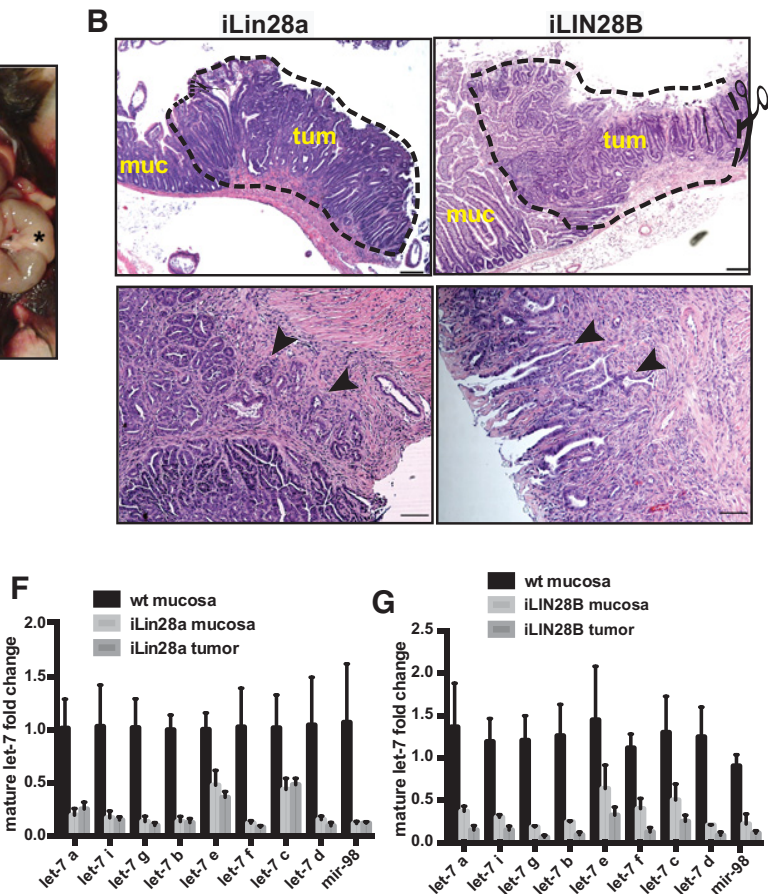

G $\quad$ wt mucosa

$2.57 \quad$ iLIN28B tumo

I
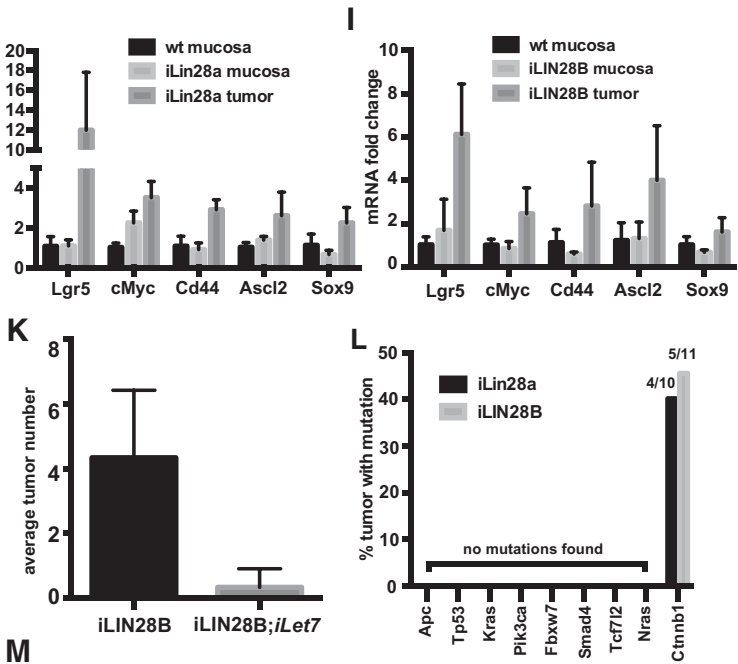

\begin{tabular}{|ccccc|}
\hline Sample name & Gene & $\begin{array}{c}\text { heterozygous allelic } \\
\text { frequencies (\%) }\end{array}$ & $\begin{array}{c}\text { Nucleotide } \\
\text { change }\end{array}$ & $\begin{array}{c}\text { Amino acid } \\
\text { change }\end{array}$ \\
\hline iLin28a T\#1 & Ctnnb1 & 22.2 & c.94 G $>$ A & p.D32N \\
iLin28a T\#6 & Ctnnb1 & 20.5 & c.95 A $>$ G & p.D32G \\
iLin28a T\#8 & Ctnnb1 & 11.6 & c.98 C>T & p.S33F \\
iLin28a T\#9 & Ctnnb1 & 17.4 & c.98 C>T & p.S33F \\
iLIN28B T\#1 & Ctnnb1 & 13.4 & c.98 C>T & p.S33F \\
iLIN28B T\#5 & Ctnnb1 & 13.1 & c. 110 C>A & p.S37Y \\
iLIN28B T\#6 & Ctnnb1 & 14.7 & c.110 C>T & p.S37F \\
iLIN28B T\#7 & Ctnnb1 & 14.1 & c. 122 C>T & p.T411 \\
iLIN28B T\#10 & Ctnnb1 & 26 & c.101 G>A & p.G34E \\
\hline
\end{tabular}

Figure 2. The LIN28/let-7 pathway cooperates with Ctnnb1 mutation to drive invasive intestinal adenocarcinoma. (A) Representative image of the small intestines in wild-type (WT), iLin28a, and iLIN28B animals 6-7 mo after doxycycline. Intestinal tumors are indicated by asterisks. $(B)$ Representative H\&E images of the iLin28a and iLIN28B intestinal adenocarcinoma with flat morphology (top panel) and invasive features (bottom panel). Arrowheads indicate the invasive ends growing into the muscularis. Bars: top panel, $200 \mu \mathrm{m}$; bottom panel, $100 \mu \mathrm{m} .(C)$ Representative immunohistochemistry staining against phostpho-Stat3 (Y705) of normal mucosa and iLin28a and iLIN28B intestinal tumors. Bar, $100 \mu \mathrm{m}$. ( $D, E)$ Quantitative PCR (qPCR) analysis showing up-regulation of tumor progression genes in iLin28a and iLIN28B tumors compared with wild-type and adjacent mucosa. Data are mean $\pm \mathrm{SD} n=3-5$ from each genotype. $(F, G)$ qRT-PCR analysis measuring the levels of mature let-7 miRNAs in wild-type intestinal mucosa, iLIN28 adjacent mucosa, and iLIN28 intestinal tumors. Data are mean $\pm \mathrm{SD} ; n=3$ from each sample type. $(H, I)$ qPCR analysis showing up-regulation of Wnt target genes in iLin28a and iLIN28B tumors. Data are mean $\pm \mathrm{SD}_{;} n=3-5$ from each sample type. $(J)$ Representative immunohistochemistry staining against Ctnnb1 ( $\beta$-catenin), Cd44, and Sox9 of normal mucosa and iLin28a and iLIN28B intestinal tumors. Bar, $100 \mu \mathrm{m}$. (K) Tumor multiplicity of iLIN28B and iLIN28B; iLet-7 animals 7 mo after doxycycline. Data are mean $\pm S D ; n=3$ from each genotype, and the animals are littermates. $(L)$ Percentage of the tumors with mutations detected in the indicated genes. $(M)$ Summary of the mutant heterozygous allele frequency and mutation position in all of the tumor samples with Ctnnb1 somatic mutation. 
invasive histology, LIN28-derived tumors expressed high levels of epithelial phosphorylated Stat 3 and elevated levels of chemokine ligand $\mathrm{Cxcll}$ and metalloprotease Mmp14 (Fig. 2C-E), which are markers for invasive tumors and are often associated with metastatic CRC (Kikuchi et al. 2000; Kusaba et al. 2005; Schwitalla et al. 2013). Notably, we failed to observe distal metastasis as late as 18 mo after doxycycline. The levels of the LIN28 proteins in the tumors were comparable with those in ESCs (Supplemental Fig. 3B,C), and, importantly, the tumors expressed minimum levels of mature let-7 (Fig. 2F,G). To determine whether LIN28-mediated tumorigenesis could be antagonized by enforced coexpression of let-7, we crossed iLIN28B animals with an inducible let-7 strain (iLet-7) engineered to express a chimeric pre-1et- $7 \mathrm{~g}$ carrying the loop region of mir-21, thus rendering it refractory to LIN28 regulation (Supplemental Fig 3D; Piskounova et al. 2008; Zhu et al. 2011). Strikingly, coexpression of the engineered let-7g almost completely reversed LIN28B-mediated tumorigenesis (Fig. 2K).

The LIN28-derived intestinal tumors manifest activated Wnt signaling, as we observed nuclear/cytoplasmic translocation of the Ctnnb1 ( $\beta$-catenin) protein and elevated levels of Cd44, Sox9, and several other classic Wnt target genes in the tumors (Fig. 2H-J). However, we ruled out the possibility that LIN28 directly activates Wnt because Wnt activation was not observed in the adjacent nontumor transgenic mucosa (Fig. 2H,I; Supplemental Fig. 3E). In addition, the long tumor latency and variable tumor multiplicity suggest that LIN28 alone is not sufficient to initiate tumor formation and that spontaneous somatic mutations of other oncogenes or tumor suppressors are required to cooperate with LIN28. To test this hypothesis, we performed targeted next-generation deep sequencing analysis of the nine most frequently mutated genes in human CRC-Apc, Tp53, Kras, Pik3ca, Fbxw7, Smad4, Tcf7L2, Nras, and Ctnnb1 (The Cancer Genome Atlas Network 2012) —in 21 iLIN28 tumors ( $n=10$ for iLin28a, and $n=11$ for iLIN28B). Interestingly, four out of 10 iLin28a and five out of 11 iLIN28B tumors harbored nonsynonymous somatic mutations of the Ctnnb1 gene that resulted in amino acid alterations in codons 32, 33, 34, 37, and 41 (Fig. 2L,M), all of which have been reported to play a role in $\beta$-catenin stabilization and Wnt hyperactivation in human CRC and other cancers (Koch et al. 1999; Mirabelli-Primdahl et al. 1999). Other than Ctnnb1, we did not detect somatic mutations in the other eight genes sequenced. The heterozygous allele frequencies of Ctnnb1 in the mutated tumors ranged from $11.6 \%$ to $26 \%$ (Fig. 2M), indicating that $23.2 \%-52 \%$ of the tumor population contains activating Ctnnb1 mutation. This percentage is consistent with our quantification of the nuclear/cytoplasmic $\beta$-catenin population from immunohistochemistry analysis (Supplemental Fig. $3 F$ ), suggesting that cells stained for translocated $\beta$-catenin are most likely those carrying mutation in the Ctnnb1 genes. In stark contrast, almost all of the cells of the $A p c^{\mathrm{Min} /+}$ tumors stained for nuclear/cytoplasmic $\beta$-catenin. These data suggest that LIN28-derived tumors are heterogeneous in nature and that some tumor subpopulations do not depend on nuclear $\beta$-catenin-mediated Wnt signaling. Thus, our murine intestinal cancer model mirrors human CRC development and supports the notion that perturbation of the Wnt pathway is the prerequisite step for tumor initiation and that reactivation of LIN28 cooperates with Wnt activation to promote intestinal tumorigenesis.

\section{LIN28 promotes tumor initiation, growth, and progression in the $\mathrm{Apc}^{\mathrm{Min} /+}$ model}

To further investigate cooperation between LIN28 and the Wnt pathway, we introduced iLIN28 into the $A p c^{M i n /+}$ strain. As a previous report concluded that activated Wnt signaling up-regulates Lin28a in breast cancer cells (Cai et al. 2013), we determined the levels of Lin28a in the $A p c^{\text {Min/+ }}$ tumors and failed to detect expression (Supplemental Fig. 4A). In addition, we examined Lin28a/b status in the dextran sulfate sodium (DSS)/azoxymethane (AOM)-induced colon tumors, which manifest activated Wnt pathways (Rosenberg et al. 2009). Similar to the $A p c^{\text {Min/+ }}$ tumors, reactivation of Lin28a or Lin28b was not observed (Supplemental Fig. 4B). Furthermore, intestine-specific conditional Lin28 and Lin28b double-knockout animals are equally susceptible to DSS/AOM-induced tumorigenesis as their wild-type littermates (Supplemental Fig. 4C). Taken together, these data indicate that in the context of intestinal tumors, activated Wnt pathway signaling does not directly activate Lin28a or Lin $28 b$ expression, and Lin28a or Lin28b expression is not absolutely required for Wnt-mediated tumor initiation.

Nevertheless, induced LIN28 overexpression significantly accelerated $A p c^{M i n /+}$ disease onset (Fig. 3A,B), increased tumor incidence (Fig. 3C-E), and enhanced tumor proliferation (Fig. 3F,H,I). Interestingly, enforced coexpression of the iLet-7 allele failed to reduce total tumor incidence (Fig. 3G) yet reduced tumor size and proliferation capacity (Fig. 3H,I; Supplemental Fig. 4D). These data suggest that tumor initiation in the $A p c^{\text {Min/+ }}$ model is enhanced by LIN28 in a let-7-independent manner but that tumor growth and proliferation are enhanced through LIN28-mediated let-7 suppression. To further investigate the mechanism by which LIN28 promotes the $A p c^{\text {Min/+ }}$ tumor phenotypes, we performed gene expression profiling comparing $A p c^{M i n /+}$ tumors and iLIN28B; $A p c^{M i n /+}$ tumors. By gene set enrichment analysis (GSEA) (Subramanian et al. 2005), we detected enhanced molecular signatures related to cell cycle and WNT in iLIN28B; $A p c^{M i n /+}$ tumors (Supplemental Fig. 4E,F), which was verified in iLin28a; $A p c^{\text {Min/+ }}$ tumors (Fig. 3J,K). In addition, GSEA analysis revealed the enrichment of several tumor progression-related pathways, such as undifferentiated cancer signature in the iLIN28B; $A p c^{\mathrm{Min} /+}$ tumors (Supplemental Fig. 4G). The up-regulation of tumor progression genes was again observed in iLin28a; $A p c^{\text {min/+ }}$ tumors (Fig. 3N). Histologically, the compound iLIN28B; $A p c^{\text {Min/+ }}$ tumors were indeed more poorly differentiated, and the tumor crypts invaded more frequently into the muscularis layer then the $A p c^{M i n /+}$ tumors (Fig. $3 \mathrm{~L}, \mathrm{M}$ ). Collectively, these data demonstrate that LIN28 promotes 
A

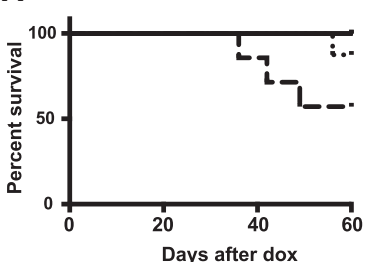

B

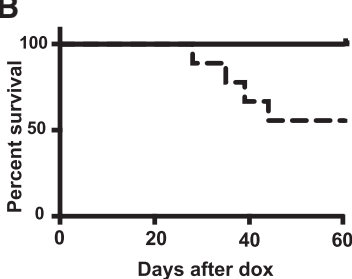

$\mathbf{F}$

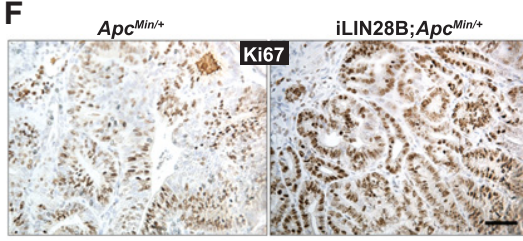

二 wt $_{\text {iLin28a }}$
C

$\therefore A p c^{\mathrm{Min} /+}$ $\rightarrow$ iLin28a; $A p c^{\mathrm{Min/4}}$

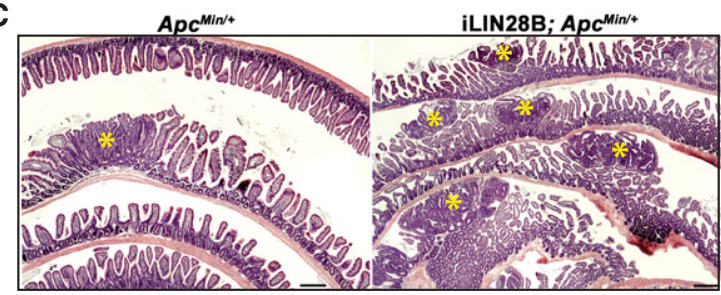

D

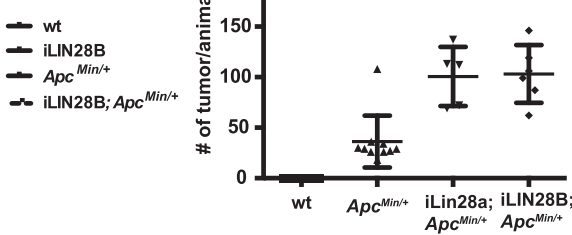

G

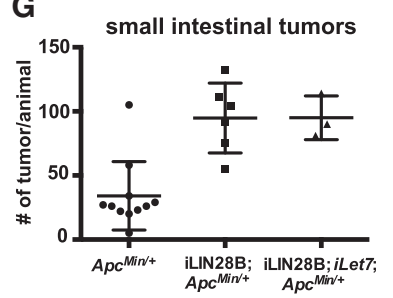

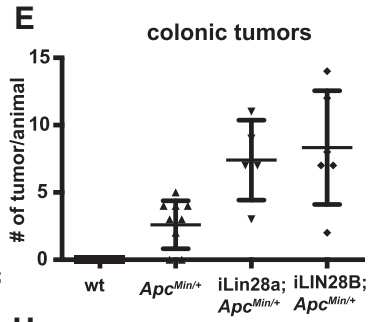

$\mathrm{H}$

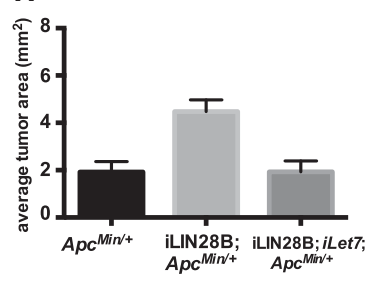

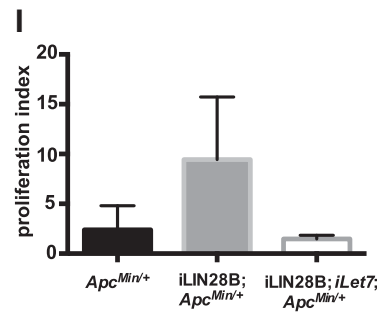
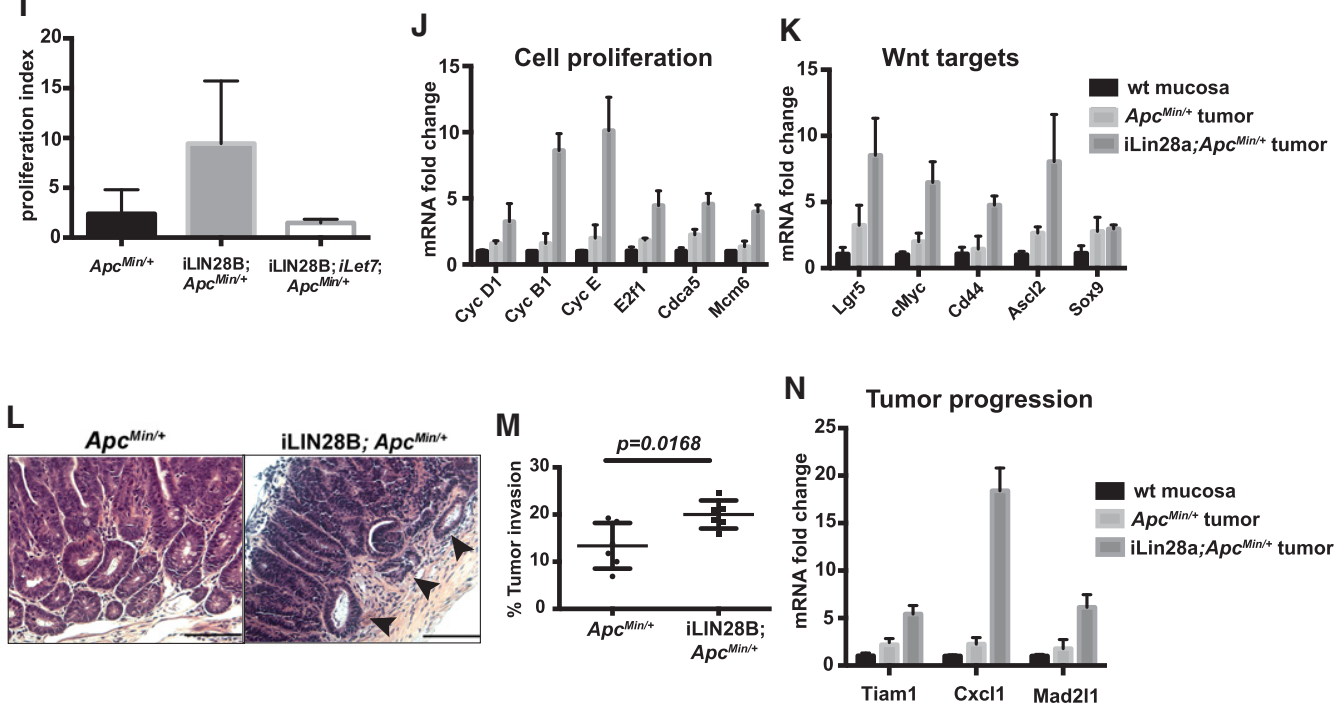

Figure 3. LIN28 promotes $A p c^{\text {Min/+ }}$ tumor initiation, growth, and progression. $(A, B)$ Kaplan-Meier curve of the indicated genotypes showing that iLin28a; $A p c^{\text {Min/+ }}$ and iLIN28B; $A p c^{M i n /+}$ animals succumbed to disease-related death faster than the $A p c^{M i n /+}$ animals. $(C)$ Representative small intestinal cross-section $\mathrm{H} \& \mathrm{E}$ images of the $A p c^{\mathrm{Min} /+}$ and iLIN28B; $A p c^{\mathrm{Min} /+}$ animals 2 mo after doxycycline treatment. Yellow asterisks indicate the tumors. Bar, $200 \mu \mathrm{m} .(D, E)$ Quantification of total intestinal tumor number and colonic tumor number developed in the indicated genotypes 2 mo after doxycycline treatment. Data are mean $\pm S D ; n=5-11$ from each genotype. $(F)$ Representative images of the $A p c^{M i n /+}$ and iLIN28B; $A p c^{M i n /+}$ small intestinal tumors stained with Mki67 antibody showing more proliferating cells in iLIN28B; $A p c^{\text {Min/+ }}$ tumors. Bar, $100 \mu \mathrm{m}$. $(G)$ Quantification of the total intestinal tumor number from each indicated genotype. Data are mean \pm SD; $n=11$ for $A p c^{M i n /+}$ animals; $n=6$ for iLIN28B; $A p c^{M i n /+}$ animals, and $n=3$ for iLIN28B; iLet-7; Apc ${ }^{\text {Min/+ }}$ animals. $(H)$ Quantification of duodenum tumor area from the indicated genotypes. Data are mean $\pm \mathrm{SD} ; n=3$ animals for each genotype; 30 tumors were randomly chosen from each animal. (I) Proliferation index of the tumors from the indicated genotypes. Data are mean \pm SD; $n=3$ animals from each genotype; three tumors from each animal. $(J, K)$ qRT-PCR analysis showing up-regulation of cell cycle genes and Wnt targets in iLin28a; $A p c^{M i n /+}$ tumors compared with $A p c^{M i n /+}$ tumors. $(L)$ Representative H\&E image of the small intestinal tumors of $A p c^{M i n /+}$ and iLIN28B; $A p c^{M i n /+}$ tumors. The iLIN28B; $A p c^{M i n /+}$ tumors invaded into the muscularis, indicated by arrowheads. Bar, 100 $\mu \mathrm{m}$. (M) Quantification of the percentage of tumors showing the invasion morphology. Data are mean $\pm \mathrm{SD} ; n=5$ from each genotype. $(N)$ qRT-PCR analysis showing up-regulation of tumor progression related genes in iLin28a; $A p c^{\text {Min/+ }}$ tumors compared with $A p c^{\text {Min/+ }}$ tumors. Data are mean $\pm \mathrm{SD} ; n=3$ from each genotype. 


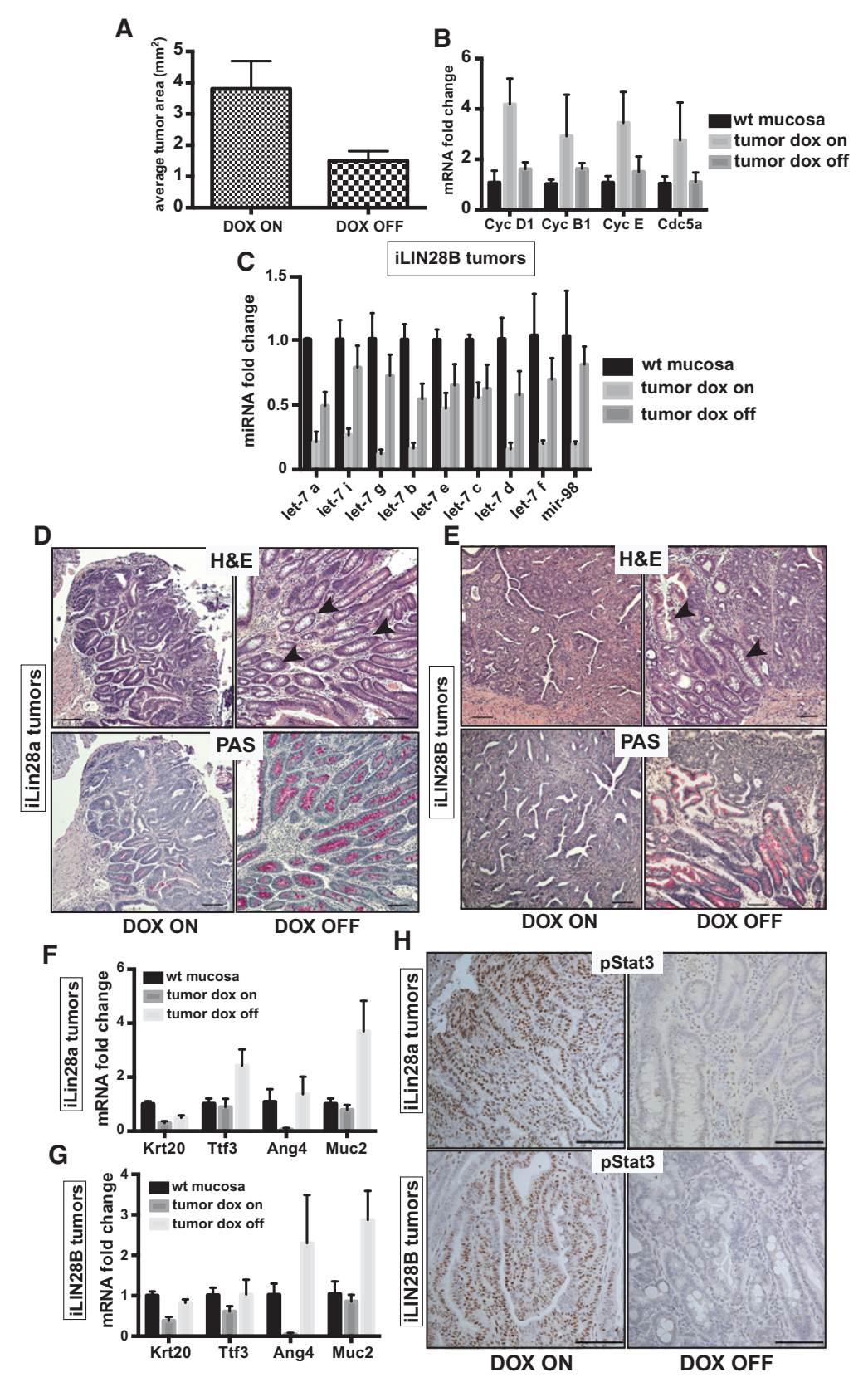

Figure 4. LIN28 sustains tumor proliferation and dedifferentiation to promote tumor progression. $(A)$ Removal of LIN28B results in decreased tumor size. Data are mean $\pm S D ; n=24$ tumors for Dox On and $n=11$ tumors for Dox Off. (B) qRT-PCR of cell cycle genes indicating that removing LIN28B expression decreases tumor proliferation. Data are mean $\pm S D$; $n=3-5$ from each sample type. $(C)$ qRT-PCR of the mature let-7 miRNAs in iLIN28B tumors with or without doxycycline withdrawn. Data are mean \pm SD; $n=3-6$ from each sample type. $(D, E)$ LIN28 expression is required for tumor dedifferentiation. Representative H\&E- and PAS-staining images showing tumor cell differentiation into goblet cells in the doxycycline-withdrawn tumors. Bar, $100 \mu \mathrm{m} .(F, G)$ qRTPCR of differentiation markers trefoil factor 3 (Tff3), keratin 20 (Krt20), angiogenin 4 (Ang4), and mucin 2 (Muc2) in the indicated sample types, showing that removal of Lin28a or LIN28B results in tumor cell differentiation into the goblet cell lineages. Data are mean $\pm \mathrm{SD} ; n=3-6$ from each sample type. $(H)$ LIN28 expression sustains tumor invasiveness. Representative immunohistochemistry against phospho-Stat3 (Y705) of iLin28a and iLIN28B tumors with or without doxycycline withdrawn. Bar, $100 \mu \mathrm{m}$.
$A p c^{\text {Min/+ }}$ tumor growth and progression and establish cooperativity between the LIN28 and Wnt pathways.

\section{LIN28 sustains tumor proliferation and dedifferentiation}

We next investigated whether LIN28 was required for tumor maintenance in vivo by withdrawing doxycycline from the iLIN28 animals after 6 mo of exposure. Two months following cessation of doxycycline, we observed reduced tumor multiplicity in both iLin28a and iLIN28B animals (Supplemental Fig. 5A). Doxycycline withdrawal in iLIN28B animals led to reduced tumor volumes and decreased proliferation markers (Fig. 4A,B). However, we did not observe shrinkage in tumor size or decreased levels of proliferation genes in iLin28a tumors after doxycycline re- moval (Supplemental Fig. 5B,C) despite the equally efficient reduction of the transgenes (Supplemental Fig. 5D, E). Interestingly, we noticed that LIN28B reduction reversed let-7 repression to a greater extent than Lin28a reduction (Fig. 4C; Supplemental Fig. 5F), suggesting that maintaining low levels of mature let-7 is important for tumor proliferation and growth.

In addition to reducing tumor growth, reduction of LIN28 transgene expression resulted in the appearance of clusters of cells with periodic acid-Schiff (PAS)-positive vacuoles, which were not seen in the tumors before doxycycline withdrawal. Moreover, doxycycline removal resulted in higher levels of several goblet cell markers, indicating induction of goblet cell differentiation when LIN28 is no longer present (Fig. 4D-G). As a poorly 
differentiated state is often associated with invasive tumor character, we observed a dramatic decrease of phospho-Stat3-expressing cells after doxycycline removal (Fig. 4H). Notably, removal of LIN28 did not reduce the levels of key Wnt target genes (Supplemental Fig. 5G,H), further indicating that LIN28 does not directly regulate Wnt in the tumors and that Wnt is independent of LIN28 status. In summary, these data suggest that sustained LIN28 expression maintains tumor cells in an undifferentiated state and promotes tumor invasiveness.
LIN28A and LIN28B are overexpressed in human CRC and are associated with invasive CRC pathology

We evaluated the expression of LIN28A and LIN28B in a cohort of 595 colon and rectal carcinoma samples using immunohistochemistry. High levels of LIN28A and/or LIN28B occurred in $38 \%$ of the samples $\left(10 \%\right.$ LIN2 $^{+} \mathrm{A}^{+}$, $8 \%$ LIN2 $^{+} \mathrm{B}^{+}$, and $20 \%$ LIN28A ${ }^{+} \mathrm{B}^{+}$) (Fig. 5A,B). Consistent with the pathology of our murine tumors, high LIN28A or LIN28B correlated with more infiltrative histology,

A

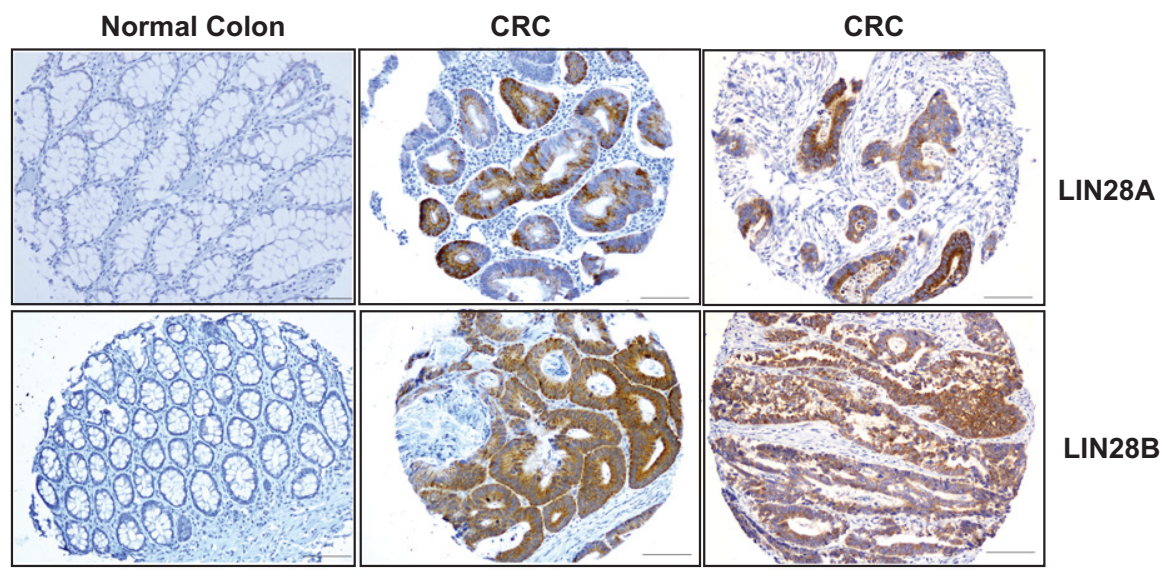

B

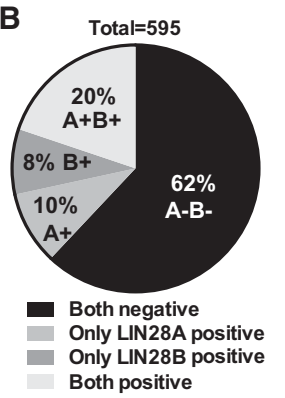

C

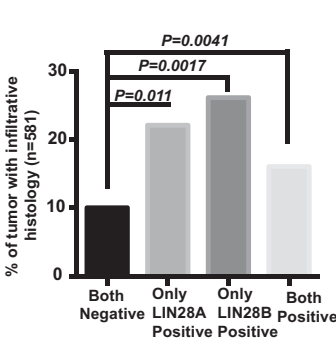

D

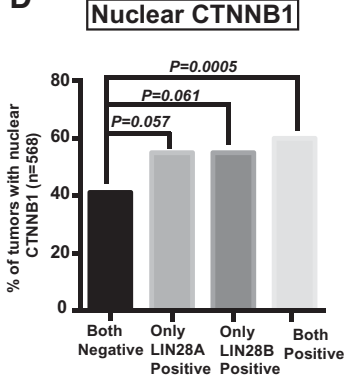

E

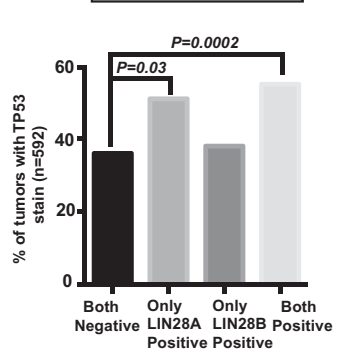

$\mathbf{F}$

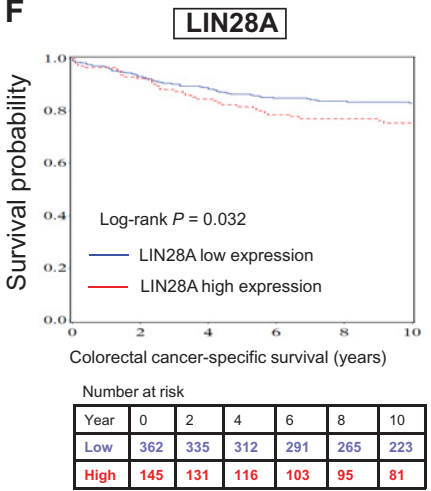

G LIN28B

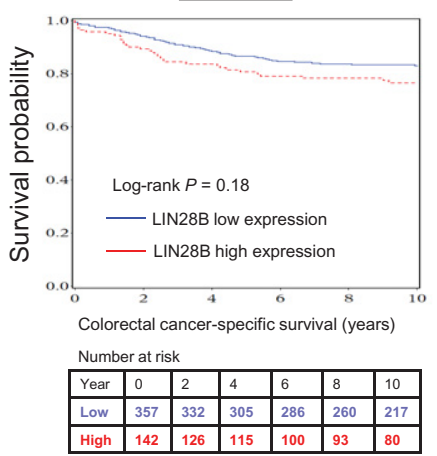

Figure 5. LIN28A and LIN28B are overexpressed in human CRC and are associated with invasive CRC pathology. (A) Representative images of LIN28A and LIN28B immunohistochemistry staining scored as "positive" samples. Bar, $100 \mu \mathrm{m}$. $(B)$ Percent distribution of the LIN28A/B status from 595 CRC cases. (C) Percent distribution of tumors with infiltrative histology according to LIN28A/B status from 581 CRC samples. $(D)$ Percent distribution of tumors with nuclear CTNNB1 stain according to LIN28A/B status from 568 CRC samples. (E) Percent distribution of tumors with positive TP53 staining according to LIN28A/B status from 592 CRC samples. (F,G) KaplanMeier curve of the 10-yr survival probabilities of CRC patients grouped according to LIN28A $(F)$ or LIN28B $(G)$ status. 
nuclear CTNNB1 staining, and positive TP53 staining (Fig. 5C-E), all of which are parameters associated with invasive tumor pathology. We further assessed the association of LIN28A and LIN28B expression with survival in stage I-III CRC and found that high expression of LIN28A was significantly associated with worse CRCspecific survival, while the association of high LIN28B expression did not reach statistical significance (Fig. 5F,G). LIN28 expression was not significantly associated with prognosis in stage IV CRC (data not shown). It is worth noting that LIN28A or LIN28B expression predicted survival probability better than KRAS, BRAF, or PIK3CA mutation in the same cohort of samples (Imamura et al. 2012; Liao et al. 2012b; Lochhead et al. 2013). Taken together, our analysis demonstrates strong association of the LIN28 proteins with invasive tumor progression and poor prognosis and provides evidence for LIN28 as a prognostic tumor biomarker for CRC.

\section{Discussion}

Here we report the generation of a robust murine model of invasive CRC, demonstrating that both LIN28A and LIN28B promote intestinal and colorectal tumorigenesis, growth, and invasive progression. Furthermore, we analyzed the largest cohort of human CRC samples to date and observed that LIN28A and/or LIN28B expression correlated with invasive tumor pathology and poor prognosis, phenotypes accurately reflected in our murine model. While prior murine models have used LIN28B exclusively (King et al. 2011a,b; Madison et al. 2013; Pang et al. 2014), we demonstrated an equally potent role of LIN28A in tumorigenesis and progression in both our murine model and human CRC.

Our models illuminate several novel aspects of LIN28induced tumor biology. While an earlier study concluded that LIN28 activation alone can drive intestinal tumorigenesis in the absence of canonical genetic alterations (Madison et al. 2013), we show that aberrant LIN28 expression results in widespread alterations in intestinal epithelial proliferation and differentiation but only focal tumor formation after a long latency, indicating a need for cooperating oncogenic events in intestinal tumor formation. Indeed, we found that a high percentage of tumors harbored mutations in the Ctnnb1 gene, indicating that spontaneous somatic mutation is a frequent mechanism by which the Wnt pathway becomes activated in our LIN28 model. Moreover, when LIN28 was induced in the $A p c^{M i n /+}$ strain, which typically develops primarily benign adenomas, tumors formed rapidly and demonstrated markedly more invasive histology, strongly suggesting that WNT pathway activation remains the tumor-initiating event, while aberrant LIN28 activation represents a driver of tumor progression and invasive character. Further supporting its role in promoting tumor progression and maintenance, withdrawal of transgenic LIN28 expression resulted in marked tumor differentiation, decreased invasiveness, and reduced tumor burden. While rare tumors have been reported with translocations involving
LIN28B, and LIN28 is included as an amplified locus in a small percentage $(1 \%-3 \%)$ of cancers /Viswanathan et al. 2009), in most cases, LIN28 overexpression has not been linked to mutation, suggesting that LIN28 is not a classical "mut driver" oncogene but rather an "epi driver" according to a recent classification of the cancer genome landscape (Vogelstein et al. 2013).

Intestinal epithelial induction of Lin28a or LIN28B resulted in partially overlapping but also independent phenotypes, implying distinct functions for the two paralogs. In the murine model, both iLin28a and iLIN28B tumors developed with a similar latency of 6 mo after doxycycline induction, and both iLin28a and iLIN28B tumors expressed high levels of Wnt target genes. However, a much higher percentage of LIN28B-induced tumors developed into high-grade adenocarcinomas compared with LIN28A-induced tumors, suggesting that the two paralogs function similarly in promoting tumor initiation but that LIN28B is more potent in conferring features of aggressive malignancy (Supplemental Fig. 3A). Moreover, although suppressing let-7 maturation is the most established molecular function of the LIN28 paralogs, only certain aspects of the tumorigenic phenotypes are opposed by enforced coexpression of let-7. Whereas in the setting of LIN28 overexpression, coexpression of let-7 reduced tumor initiation, when LIN28 was overexpressed in the $A p c^{\text {Min/+ }}$ background, coexpression of let-7 had no effect on tumor incidence but did reduce tumor proliferation. One possibility is that in the $A p c^{M i n /+}$ animals, LIN28 promotes tumor initiation by increasing the chance of $A p c$ loss of heterozygosity (LOH), while in the iLIN28 model, LIN28 promotes tumor initiation by expanding the highly proliferative and undifferentiated crypt cells. The distinct molecular targets regulated by LIN2 8 to promote tumor development in different models await further investigation. The development of intestinal rather than colonic tumors in our murine model is similar to what is commonly reported in many other GI tumor murine models. A recent report suggests that the incidence of tumor formation is largely correlated with the number of stem cells residing in the respective tissues and that mouse small intestines contain higher numbers of cycling stem cells than mouse colons (Tomasetti and Vogelstein 2015).

The important roles of LIN28/let-7 in tumor progression implicate this pathway as an attractive therapeutic target for CRC. Normal adult intestinal epithelium does not express either paralog, and intestine-specific Lin28a/ Lin28b double-knockout animals have no obvious intestinal phenotype (H-C Tu and GQ Daley, unpubl.). These observations indicate that targeting the LIN28 pathway would have minimal effects on normal intestinal homeostasis. Importantly, we demonstrated here that reversal of LIN28 expression in a full-blown tumor produces tumor cell differentiation and reduced tumor invasiveness. These data agree with our hypothesis that intestinal tumor cells use an embryonic molecular signature to maintain cells in an undifferentiated state. We propose that antagonizing LIN28 would induce tumor cell differentiation and might prove beneficial in combination with chemotherapy, as well-differentiated tumors are typically 
less aggressive and less drug-resistant and have better clinical outcomes. Last, most current mouse models for CRC, including the $A p c^{\mathrm{Min} /+}$ model, fail to manifest invasive cancer and thus fail to accurately reflect the pathology and natural history of late-stage CRC. Our transgenic strains of iLIN28 mice represent robust and reliable models of late-stage CRC that closely mimic human disease pathologically and molecularly and should prove useful as in vivo preclinical platforms for developing therapeutics.

\section{Materials and methods}

Mice

All animal procedures were conducted according to animal care guidelines approved by the Institutional Animal Care and Use Committee at Boston Children's Hospital. Lox-stop-Lox-TetOnLin28 and conditional Lin28a and Lin28b double-knockout mice were previously generated in our laboratory (Zhu et al. 2011; Shinoda et al. 2013). Villin-Cre (stock no. 004586) and $A p c^{\text {Min/+ }}$ (stock no. 002020) animals were obtained from Jackson Laboratory. For transgene induction, $1 \mathrm{~g} / \mathrm{L}$ doxycycline (Sigma) was administered to the drinking water at different time points to induce LIN28B and Lin28 a transgenes. To achieve co-overexpression of Lin28 and Let7, we crossed Villin-cre mice with TRE-7S21L ("iLet-7") (described in Zhu et al. 2011) mice and then crossed the Villin-cre; iLet-7 mice with Lox-stop-Lox-TetOn-LIN28 mice.

\section{Histology and immunohistochemistry}

Swiss-rolled intestinal tumor and normal tissues were fixed in $10 \%$ buffered formalin and embedded in paraffin. Immunostaining was performed using the following antibodies: anti-Cdx2 (MU392A-UC, Biogenex ), anti-Lin28a (\#3978, Cell Signaling), anti-LIN28B (\#4196, Cell Signaling), anti- $\beta$-catenin (\#610154, BD Transduction), anti-Cd44 (\#550538, BD Pharmingen ), antiStat3 (phosphorylated at Y705) (\#9145, Cell Signaling), antiSox9 (AB5535, Millipore), anti-Lyz (lysozyme) (A0099, Dako ), and anti-Mki67 (M7249, Dako ). Slides were dewaxed with xylene and rehydrated through a series of washes with decreasing percentages of ethanol. Antigen retrieval was performed in $10 \mathrm{mM}$ sodium citrate buffer ( $\mathrm{pH}$ 6.0) by placement in a decloaking chamber for $30 \mathrm{~min}$ at $95^{\circ} \mathrm{C}$. Immunohistochemistry was performed with Elite $\mathrm{ABC}$ kit and $\mathrm{DAB}$ substrate (Vector Laboratories) according to the manufacturer's protocol. PAS staining was used to detect neutral and acid mucins and mucus substances according to standard procedures.

\section{Quantification of proliferation index, tumor sizes,} and invasiveness

The proliferation index is defined as the number of Mki67-positive cells over the number of Mki67-negative cells per crypt or per tumor. Thirty crypts were calculated per animal, and three to five animals per genotype were analyzed for the crypts. Three tumors per animal and three to five animals per genotype were analyzed for the tumors. Tumor sizes were measured by using the Nikon Eclipse 90i microscope and NIS-Elements Viewer software (Nikon). Tumor invasiveness was analyzed by reviewing the histology for tumors with incomplete basal membrane and the presence of irregular-shaped tumor crypts invading into the muscularis.

\section{Quantitative RT-PCR ( $q R T-P C R)$}

RNA was isolated by TRIzol and reverse-transcribed using a miScript II RT kit (catalog no. 218161, Qiagen). mRNA expression was measured by qPCR using the $\triangle \triangle \mathrm{CT}$ method with the following primers: mLgr5 (forward primer, $5^{\prime}$-GACAATGCTCTCACA GAC-3'; reverse primer, 5'-GGAGTGGATTCTATTATTATGG $-3^{\prime}$ ), mcMyc (forward primer, 5'-CTTACAATCTGCGAGCCAG GACA-3'; reverse primer, 5'-GCTGTACGGAGTCGTAGTC GAG-3'), mCd44 (forward primer, 5'-CACCATTGCCTCAA CTGTGC-3'; reverse primer, 5'-TTGTGGGCTCCTGAGTC TGA-3'), mAscl2 (forward primer, 5'-CTACTCGTC GGAG GAAAG-3'; reverse primer, 5'-ACTAGACAGCATGGGTAAG$3^{\prime}$ ), mCyclin D1 (forward primer, 5'-CGTATCTTACTTCAA GTGCGTGCAGAAG-3'; reverse primer, 5'-CAGGTTCCACTT GAGCTTGTTCAC- $3^{\prime}$ ), mCyclin B1 (forward primer, $5^{\prime}$-ACTT CAGCCTGGGTCGCC-3'; reverse primer, 5'-ACGTCAAC CTCTCCGACTTTAGA-3'), mCyclin E1 (forward primer, 5'ATGTGGCCGTGTTTTGCA-3'; reverse primer, 5'-GGTCTG ATTTTCCGAGGCTGA-3'), mE2f1 (forward primer, $5^{\prime}$-GGAT CTGGAGACTGACCATCAG-3'; reverse primer, 5' -GGTTTCA TAGCGTGACTTCTCCC-3'), mMcm6 (forward primer, 5'-CG ACAGCTTGAGAGCATGATCC-3'; reverse primer, 5'-TGAC ATCAGGCGTCTCTACACG-3'), mCdca5 (forward primer, 5'AGTCGTCCAAGCGGAAATCTGG-3'; reverse primer, 5'-CC TCTACTGCATGAGCTACGATC-3') , mSox9 (forward primer, 5'-GCAGACCAGTACCCGCATCT-3'; reverse primer, 5'-TCT CGTTCAGCAGCCTCCA-3'), mMmp14 (forward primer, 5'-GGATGGACACAGAGAACTTCGTG-3'; reverse primer, 5'CGAGAGGTAGTTCTGGGTTGAG-3'), mCxcl1 (forward primer, 5'-TATCGCCAATGAGCTGCG-3'; reverse primer, 5'-GG ATGTTCTTGAGGTGAATCCC-3'), mMad211 (forward primer, 5'-CAGAAACTGGTGGTGGTCATCTC-3'; reverse primer, 5'-CATCCTGTATGGCTTTCTGGGAC-3'), mTiam1 (forward primer, 5'-CCACATTGAGAAGTCAGACGCG-3'; reverse primer, 5' -CGGTTTCCTTGACACTGTTCACG-3'), mKrt20 (forward primer, 5'-CCCAGAAGAACCTGCAAGAG-3'; reverse primer, 5'-ACGAGCCTTGACGTCCTCTA-3'), mTtf3 (forward primer, 5'-TCCAAGCCAATGTATGGTGCCG-3'; reverse primer, 5'CAGGGCACATTTGGGATACTGG-3'), mAng4 (forward primer, 5'-GGCACCAAGAAAAACATCAGGGC-3' ; reverse primer, 5'-GTGCGTACAAGTGGTGATCTGG-3'), and mMuc2 (forward primer, 5'-GGTCCAGGGTCTGGATCACA-3'; reverse primer, 5'-GCTCAGCTCACTGCCATCTG-3'). For qRT-PCR of mature let-7 miRNA, we used Qiagen miScript target as described in the miScript protocol.

\section{Western blot}

Cells were lysed in RIPA buffer (Pierce) supplemented with protease inhibitor cocktail (Roche) and phosphatase inhibitor cocktail (Roche). Proteins was separated by a $12 \%$ or $4 \%-12 \%$ polyacrylamide gel (Bio-Rad) and transferred to a methanol-activated PVDF membrane (GE Healthcare). The membrane was blocked for $1 \mathrm{~h}$ in PBST containing 6\% milk and subsequently probed with primary antibodies overnight at $4^{\circ} \mathrm{C}$. After 1 -h incubation with sheep anti-mouse or donkey anti-rabbit HRP-conjugated secondary antibody (GE Healthcare), the protein level was detected with standard ECL reagents (Thermo Scientific). Antibodies used were anti- $\alpha / \beta$-tubulin (\#2148, Cell Signaling), antiActb ( $\beta$-actin) (sc-69879, Santa Cruz Biotechnology), anti-Cdx2 (MU392A-UC, Biogenex), anti-Lin28a (\#3978, Cell Signaling), anti-LIN28B (\#4196, Cell Signaling), anti-Lin28b (mouse preferred) (\#5422, Cell Signaling), anti-Flag (M2 clone, Sigma), anti-Cyclin D1 (ab10540, Abcam), and anti-Sox 9 (AB5535, Millipore). 


\section{Microarray analysis}

RNA from two duodenum tumors from $\mathrm{Apc}^{\mathrm{Min} /+}$ and iLIN28; $A p c^{M i n /+}$ littermates were harvested and processed using TRIzoL. The Illumina Ref- 8 microarray platform was used by the Boston Children's Hospital Intellectual and Developmental Disabilities Research Center (IDDRC) Molecular Genetics Core Facility. The microarray data have been deposited in Gene Expression Omnibus (GEO) and given the series accession number GSE68334. GSEA (http://www.broadinstitute.org/gsea/index.jsp) was used to identify gene sets and pathways associated with a set of up-regulated or down-regulated genes.

\section{DSS/AOM protocol}

DSS (molecular weight 36,000-50,000 g/mol) was obtained from MP Biomedicals, and AOM was obtained from Sigma (catalog no. A5486). Six-week-old to 8-wk-old C57/BL6 wild-type animals were given a dose of $10 \mathrm{mg} / \mathrm{kg}$ AOM intraperitoneally. One week later, mice received $2.5 \%$ DSS in the drinking water for $5 \mathrm{~d}$ followed by 2 wk of water. Two cycles of DSS were administered, animals were sacrificed at day 55 after AOM treatment, and colon tumors were harvested for protein analysis. Lin28 $a^{\text {flox } /-}$; Lin28 $b^{\text {flox/-}}$;Villin-Cre ${ }^{+}$(Lin28a/b double knockout) and littermate control Lin28 $a^{\text {flox } /+}{ }_{;}$Lin28 $b^{\text {flox } /+}{ }_{;}$Villin-Cre ${ }^{+}$(wild type) were in mixed background (C57BL6/129sv/Balbc); due to the background-dependent susceptibility to DSS, the protocol was modified to three cycles of $3.5 \%$ DSS, with $7 \mathrm{~d}$ of DSS treatment in each cycle. Animals were sacrificed at $66 \mathrm{~d}$ after AOM treatment.

\section{Targeted next-generation DNA sequencing}

Amplicons were designed for nine genes of interest by Ion AmpliSeq Designer (Life Technologies). Libraries were made with $10 \mathrm{ng}$ of genomic DNA following ion AmpliSeq library kit 2.0 protocol. These libraries were then templated using Ion Onetouch 2 (Life Technologies) and sequenced on an Ion 318 chip (Life Technologies) following the manufacturer's instructions. Data from the PGM runs were analyzed using Torrent Suite software to generate sequencing reads and perform variant calling (custom somatic high-stringency parameters with allele frequency $>10 \%)$. These variants were annotated with ANNOVAR (Wang et al. 2010) using mouse $\mathrm{mm} 10$ genome assembly and curated by literature reviews.

\section{Statistical tests}

Data expressions are mean \pm SD. Differences were analyzed by Student's $t$-test using Prism 6 (GraphPad Prism software).

\section{Human CRC samples}

We used the database of two United States nationwide prospective cohort studies: the Nurses' Health Study (NHS; $N=$ 121,700 women observed since 1976) and the Health Professionals Follow-Up Study (HPFS; $N=51,500$ men observed since 1986) (Liao et al. 2012a). Participants were sent follow-up biennial questionnaires to update information on diet and lifestyle factors and identify newly diagnosed cancers and other diseases. We collected paraffin-embedded tissue blocks from hospitals where participants with CRC had undergone resection of primary tumors. All CRC cases were confirmed through review of histology by a pathologist (S. Ogino) blinded to exposure data. Tumor grade was categorized as high $(\leq 50 \%$ glandular area) or low $(>50 \%$ glandular area). The type of tumor growth pattern at the tumor margin was examined at low-power magnification and categorized as expansile, intermediate, or infiltrative (Morikawa et al. 2012). Diagnostic biopsy specimens from rectal cancer patients who received preoperative therapy were collected in order to avoid treatmentrelated artifacts. Patients were observed until death or January 2013, whichever came first. Written informed consent was obtained from all study subjects. The human subject committees at Harvard School of Public Health and Brigham and Women's Hospital approved this study.

Immunohistochemistry for LIN28A, LIN28B, TP53, and CTNNB1 in human samples

Tissue microarray blocks were constructed as previously described (Chan et al. 2009). Methods of immunohistochemical staining and interpretations for TP53 and CTNNB1 have been described previously (Baba et al. 2011; Morikawa et al. 2011). For LIN28A and LIN28B immunostaining, deparaffinized tissue sections were heated in a microwave for $15 \mathrm{~min}$ in antigen retrieval citra solution at $\mathrm{pH} 6$ (BioGenex Laboratories). Tissue sections were incubated with dual endogenous enzyme block (DAKO) and then serum-free protein block (DAKO) for 15 min each. Slides were incubated for $16 \mathrm{~h}$ at $4^{\circ} \mathrm{C}$ with a primary antibody against LIN28A (1:50, rabbit monoclonal; no. 8641, Cell Signaling) or LIN28B (1:75, rabbit polyclonal; no. 4196, Cell Signaling). Envision anti-rabbit HRP-labeled polymer (DAKO) was applied to the sections for $30 \mathrm{~min}$ followed by visualization using the chromogen 3,3-diaminobenzidine (DAKO) and hematoxylin counterstain. Positive and negative controls were included in each panel of immunohistochemistry for all markers. Each immunohistochemical marker was evaluated by a pathologist (Z Qian) unaware of other data.

\section{Statistical analysis for human samples}

All statistical analyses were performed with the SAS program (version 9.1, SAS Institute). All $P$-values were two-sided, and statistical significance was set at $P=0.05$. For categorical data, the $\chi^{2}$ test was performed. The Kaplan-Meier method and log-rank test were used for survival analyses. Deaths from causes other than CRC were censored in CRC-specific mortality analyses. To control for confounding, we used Cox proportional hazard models to calculate the hazard ratio (HR) of death according to tumor LIN28A or LIN28B expression status. The model initially included age at diagnosis (continuous), sex, body mass index, tumor location (proximal vs. distal colon vs. rectum), TMN stage, tumor grade, MSI (high vs. low/MSS), CIMP (high vs. low/0), LINE-1 methylation (continuous), BRAF mutation, KRAS mutation, and PIK3CA mutation in addition to CTNNB1, phosphorylated AKT, and DNMT1 expression. To minimize residual confounding and overfitting, disease stage (I, II, III, IV, or unknown) was used as a stratifying variable using the "strata" option in the SAS "proc phreg" command. To avoid overfitting, variables in the final model were selected using backward stepwise elimination with a threshold of $P=0.05$.

\section{Acknowledgments}

We thank Achia Urbach, John Powers, Ramesh Shivdasani, and $\mathrm{Xi}$ He for invaluable discussion and advice. We thank Michael C.F. Chen for providing E12.5 embryonic intestine tissues, and Roderick Bronson and the Harvard Medical School Rodent Histopathology Core for mouse tissue pathology. G.Q.D. is supported by grants from the National Institutes of Health $\left(\mathrm{NIH}_{\text {; }}\right.$ 
R01GM107536) and the Ellison Medical Foundation and is an affiliate member of the Broad Institute and an investigator of the Howard Hughes Medical Institute and the Manton Center for Orphan Disease Research. H.-C.T. is supported by the American Association for Cancer Research Fight Colorectal Cancer Postdoctoral Fellowship. S.S. is supported by an EMBO post-doctoral fellowship. A.Y. is supported by NIH F31 CA186444-01 predoctoral fellowship. R.N. is supported by NIH K07 CA190673. C.S. F. is supported by NIH P01CA127003 and Agrusa Fund for Colorectal Cancer Research. S.O. is supported by NIH RO1CA151993 and DFIC Friends.

\section{References}

Ambros V, Horvitz HR. 1984. Heterochronic mutants of the nematode Caenorhabditis elegans. Science 226: 409-416.

Baba Y, Nosho K, Shima K, Hayashi M, Meyerhardt JA, Chan AT, Giovannucci E, Fuchs CS, Ogino S. 2011. Phosphorylated AKT expression is associated with PIK3CA mutation, low stage, and favorable outcome in 717 colorectal cancers. Cancer 117: 1399-1408.

Beachy SH, Onozawa M, Chung YJ, Slape C, Bilke S, Francis P, Pineda M, Walker RL, Meltzer P, Aplan PD. 2012. Enforced expression of Lin28b leads to impaired T-cell development, release of inflammatory cytokines, and peripheral T-cell lymphoma. Blood 120: 1048-1059.

Ben-Porath I, Thomson MW, Carey VI, Ge R, Bell GW, Regev A, Weinberg RA. 2008. An embryonic stem cell-like gene expression signature in poorly differentiated aggressive human tumors. Nat Genet 40: 499-507.

Blaker H, Scholten M, Sutter C, Otto HF, Penzel R. 2003. Somatic mutations in familial adenomatous polyps. Nuclear translocation of $\beta$-catenin requires more than biallelic APC inactivation. Am J Clin Pathol 120: 418-423.

Brabletz T, Jung A, Reu S, Porzner M, Hlubek F, Kunz-Schughart LA, Knuechel R, Kirchner T. 2001. Variable $\beta$-catenin expression in colorectal cancers indicates tumor progression driven by the tumor environment. Proc Natl Acad Sci 98: 1035610361.

Bussing I, Slack FJ, Grosshans H. 2008. let-7 microRNAs in development, stem cells and cancer. Trends Mol Med 14: 400-409.

Cai WY, Wei TZ, Luo QC, Wu QW, Liu QF, Yang M, Ye GD, Wu JF, Chen YY, Sun GB, et al. 2013. The Wnt- $\beta$-catenin pathway represses let-7 microRNA expression through transactivation of Lin28 to augment breast cancer stem cell expansion. J Cell Sci 126: 2877-2889.

The Cancer Genome Atlas Network. 2012. Comprehensive molecular characterization of human colon and rectal cancer. Nature 487: 330-337.

Chan AT, Ogino S, Fuchs CS. 2009. Aspirin use and survival after diagnosis of colorectal cancer. JAMA 302: 649-658.

Clevers H. 2013. The intestinal crypt, a prototype stem cell compartment. Cell 154: 274-284.

Diskin SJ, Capasso M, Schnepp RW, Cole KA, Attiyeh EF, Hou C, Diamond M, Carpenter EL, Winter C, Lee H, et al. 2012. Common variation at 6q16 within HACE1 and LIN28B influences susceptibility to neuroblastoma. Nat Genet 44: 1126-1130.

Fearon ER. 2011. Molecular genetics of colorectal cancer. Annu Rev Pathol 6: 479-507.

Fearon ER, Vogelstein B. 1990. A genetic model for colorectal tumorigenesis. Cell 61: 759-767.

Fodde R, Brabletz T. 2007. Wnt $/ \beta$-catenin signaling in cancer stemness and malignant behavior. Curr Opin Cell Biol 19: $150-158$.
Gillis AJ, Stoop H, Biermann K, van Gurp RJ, Swartzman E, Cribbes S, Ferlinz A, Shannon M, Oosterhuis JW, Looijenga LH. 2011. Expression and interdependencies of pluripotency factors LIN28, OCT3/4, NANOG and SOX2 in human testicular germ cells and tumours of the testis. Int I Androl 34: e160-e174.

Gregorieff A, Clevers H. 2005. Wnt signaling in the intestinal epithelium: from endoderm to cancer. Genes Dev 19: 877-890.

Guo Y, Chen Y, Ito H, Watanabe A, Ge X, Kodama T, Aburatani H. 2006. Identification and characterization of lin-28 homolog B (LIN28B) in human hepatocellular carcinoma. Gene 384: $51-61$.

Heo I, Joo C, Cho J, Ha M, Han J, Kim VN. 2008. Lin28 mediates the terminal uridylation of let-7 precursor MicroRNA. Mol Cell 32: 276-284.

Imamura Y, Morikawa T, Liao X, Lochhead P, Kuchiba A, Yamauchi M, Qian ZR, Nishihara R, Meyerhardt JA, Haigis KM, et al. 2012. Specific mutations in KRAS codons 12 and 13, and patient prognosis in 1075 BRAF wild-type colorectal cancers. Clin Cancer Res 18: 4753-4763.

Janssen KP, Alberici P, Fsihi H, Gaspar C, Breukel C, Franken P, Rosty C, Abal M, El Marjou F, Smits R, et al. 2006. APC and oncogenic KRAS are synergistic in enhancing Wnt signaling in intestinal tumor formation and progression. Gastroenterology 131: 1096-1109.

Johnson SM, Grosshans H, Shingara J, Byrom M, Jarvis R, Cheng A, Labourier E, Reinert KL, Brown D, Slack FJ. 2005. RAS is regulated by the let-7 microRNA family. Cell 120: 635-647.

Kikuchi R, Noguchi T, Takeno S, Kubo N, Uchida Y. 2000. Immunohistochemical detection of membrane-type-1-matrix metalloproteinase in colorectal carcinoma. Br $J$ Cancer 83: 215-218.

Kim J, Orkin SH. 2011. Embryonic stem cell-specific signatures in cancer: insights into genomic regulatory networks and implications for medicine. Genome Med 3: 75.

King CE, Cuatrecasas M, Castells A, Sepulveda AR, Lee JS, Rustgi AK. 2011a. LIN28B promotes colon cancer progression and metastasis. Cancer Res 71: 4260-4268.

King CE, Wang L, Winograd R, Madison BB, Mongroo PS, Johnstone CN, Rustgi AK. 2011b. LIN28B fosters colon cancer migration, invasion and transformation through let-7-dependent and -independent mechanisms. Oncogene 30: 4185-4193.

Kinzler KW, Vogelstein B. 1996. Lessons from hereditary colorectal cancer. Cell 87: 159-170.

Koch A, Denkhaus D, Albrecht S, Leuschner I, von Schweinitz D, Pietsch T. 1999. Childhood hepatoblastomas frequently carry a mutated degradation targeting box of the $\beta$-catenin gene. Cancer Res 59: 269-273.

Kusaba T, Nakayama T, Yamazumi K, Yakata Y, Yoshizaki A, Nagayasu T, Sekine I. 2005. Expression of p-STAT3 in human colorectal adenocarcinoma and adenoma; correlation with clinicopathological factors. J Clin Pathol 58: 833-838.

Lee YS, Dutta A. 2007. The tumor suppressor microRNA let-7 represses the HMGA2 oncogene. Genes Dev 21: 1025-1030.

Liao X, Lochhead P, Nishihara R, Morikawa T, Kuchiba A, Yamauchi M, Imamura Y, Qian ZR, Baba Y, Shima K, et al. 2012a. Aspirin use, tumor PIK3CA mutation, and colorectalcancer survival. N Engl J Med 367: 1596-1606.

Liao X, Morikawa T, Lochhead P, Imamura Y, Kuchiba A, Yamauchi M, Nosho K, Qian ZR, Nishihara R, Meyerhardt JA, et al. 2012b. Prognostic role of PIK3CA mutation in colorectal cancer: cohort study and literature review. Clin Cancer Res 18: 2257-2268.

Lochhead P, Kuchiba A, Imamura Y, Liao X, Yamauchi M, Nishihara R, Qian ZR, Morikawa T, Shen J, Meyerhardt JA, et al. 
2013. Microsatellite instability and BRAF mutation testing in colorectal cancer prognostication. I Natl Cancer Inst 105: 1151-1156.

Madison BB, Liu Q, Zhong X, Hahn CM, Lin N, Emmett MJ, Stanger BZ, Lee JS, Rustgi AK. 2013. LIN28B promotes growth and tumorigenesis of the intestinal epithelium via Let-7. Genes Dev 27: 2233-2245.

Mayr C, Hemann MT, Bartel DP. 2007. Disrupting the pairing between let-7 and Hmga2 enhances oncogenic transformation. Science 315: 1576-1579.

Mirabelli-Primdahl L, Gryfe R, Kim H, Millar A, Luceri C, Dale D, Holowaty E, Bapat B, Gallinger S, Redston M. 1999. $\beta$-Catenin mutations are specific for colorectal carcinomas with microsatellite instability but occur in endometrial carcinomas irrespective of mutator pathway. Cancer Res 59: 3346-3351.

Molenaar JJ, Domingo-Fernandez R, Ebus ME, Lindner S, Koster J, Drabek K, Mestdagh P, van Sluis P, Valentijn LJ, van Nes J, et al. 2012. LIN28B induces neuroblastoma and enhances MYCN levels via let-7 suppression. Nat Genet 44: 1199-1206.

Morikawa T, Kuchiba A, Yamauchi M, Meyerhardt JA, Shima K, Nosho K, Chan AT, Giovannucci E, Fuchs CS, Ogino S. 2011. Association of CTNNB1 ( $\beta$-catenin) alterations, body mass index, and physical activity with survival in patients with colorectal cancer. JAMA 305: 1685-1694.

Morikawa T, Kuchiba A, Qian ZR, Mino-Kenudson M, Hornick JL, Yamauchi M, Imamura Y, Liao X, Nishihara R, Meyerhardt JA, et al. 2012. Prognostic significance and molecular associations of tumor growth pattern in colorectal cancer. Ann Surg Oncol 19: 1944-1953.

Moss EG, Tang L. 2003. Conservation of the heterochronic regulator Lin-28, its developmental expression and microRNA complementary sites. Dev Biol 258: 432-442.

Moss EG, Lee RC, Ambros V. 1997. The cold shock domain protein LIN-28 controls developmental timing in C. elegans and is regulated by the lin-4 RNA. Cell 88: 637-646.

Nadiminty N, Tummala R, Lou W, Zhu Y, Shi XB, Zou JX, Chen H, Zhang J, Chen X, Luo J, et al. 2012. MicroRNA let-7c is downregulated in prostate cancer and suppresses prostate cancer growth. PLoS One 7: e32832.

Newman MA, Thomson JM, Hammond SM. 2008. Lin-28 interaction with the Let-7 precursor loop mediates regulated microRNA processing. RNA 14: 1539-1549.

Nguyen LH, Robinton DA, Seligson MT, Wu L, Li L, Rakheja D, Comerford SA, Ramezani S, Sun X, Parikh MS, et al. 2014. Lin28b is sufficient to drive liver cancer and necessary for its maintenance in murine models. Cancer Cell 26: 248-261.

Pang M, Wu G, Hou X, Hou N, Liang L, Jia G, Shuai P, Luo B, Wang K, Li G. 2014. LIN28B promotes colon cancer migration and recurrence. PLoS One 9: e109169.

Piskounova E, Viswanathan SR, Janas M, LaPierre RJ, Daley GQ, Sliz P, Gregory RI. 2008. Determinants of microRNA processing inhibition by the developmentally regulated RNA-binding protein Lin28. J Biol Chem 283: 21310-21314.

Piskounova E, Polytarchou C, Thornton JE, LaPierre RJ, Pothoulakis C, Hagan JP, Iliopoulos D, Gregory RI. 2011. Lin28A and Lin28B inhibit let-7 microRNA biogenesis by distinct mechanisms. Cell 147: 1066-1079.

Rosenberg DW, Giardina C, Tanaka T. 2009. Mouse models for the study of colon carcinogenesis. Carcinogenesis 30: $183-196$.
Sato T, van Es JH, Snippert HI, Stange DE, Vries RG, van den Born M, Barker N, Shroyer NF, van de Wetering M, Clevers H. 2011. Paneth cells constitute the niche for Lgr5 stem cells in intestinal crypts. Nature 469: 415-418.

Schwitalla S, Ziegler PK, Horst D, Becker V, Kerle I, Begus-Nahrmann Y, Lechel A, Rudolph KL, Langer R, Slotta-Huspenina J, et al. 2013. Loss of p53 in enterocytes generates an inflammatory microenvironment enabling invasion and lymph node metastasis of carcinogen-induced colorectal tumors. Cancer Cell 23: 93-106.

Shinoda G, Shyh-Chang N, Soysa TY, Zhu H, Seligson MT, Shah SP, Abo-Sido N, Yabuuchi A, Hagan JP, Gregory RI, et al. 2013. Fetal deficiency of $\operatorname{lin} 28$ programs life-long aberrations in growth and glucose metabolism. Stem Cells 31: 1563-1573.

Shyh-Chang N, Daley GQ. 2013. Lin28: primal regulator of growth and metabolism in stem cells. Cell Stem Cell 12: 395-406.

Shyh-Chang N, Zhu H, Yvanka de Soysa T, Shinoda G, Seligson MT, Tsanov KM, Nguyen L, Asara JM, Cantley LC, Daley GQ. 2013. Lin28 enhances tissue repair by reprogramming cellular metabolism. Cell 155: 778-792.

Subramanian A, Tamayo P, Mootha VK, Mukherjee S, Ebert BL, Gillette MA, Paulovich A, Pomeroy SL, Golub TR, Lander ES, et al. 2005. Gene set enrichment analysis: a knowledgebased approach for interpreting genome-wide expression profiles. Proc Natl Acad Sci 102: 15545-15550.

Taketo MM, Edelmann W. 2009. Mouse models of colon cancer. Gastroenterology 136: 780-798.

Tomasetti C, Vogelstein B. 2015. Cancer etiology. Variation in cancer risk among tissues can be explained by the number of stem cell divisions. Science 347: 78-81.

Urbach A, Yermalovich A, Zhang J, Spina CS, Zhu H, PerezAtayde AR, Shukrun R, Charlton J, Sebire N, Mifsud W, et al. 2014. Lin28 sustains early renal progenitors and induces Wilms tumor. Genes Dev 28: 971-982.

Viswanathan SR, Daley GQ, Gregory RI. 2008. Selective blockade of microRNA processing by Lin28. Science 320: 97-100.

Viswanathan SR, Powers JT, Einhorn W, Hoshida Y, Ng TL, Toffanin S, O'Sullivan M, Lu J, Phillips LA, Lockhart VL, et al. 2009. Lin 28 promotes transformation and is associated with advanced human malignancies. Nat Genet 41: 843-848.

Vogelstein B, Papadopoulos N, Velculescu VE, Zhou S, Diaz LA Jr, Kinzler KW. 2013. Cancer genome landscapes. Science 339: 1546-1558.

Wang K, Li M, Hakonarson H. 2010. ANNOVAR: functional annotation of genetic variants from high-throughput sequencing data. Nucleic Acids Res 38: e164.

West JA, Viswanathan SR, Yabuuchi A, Cunniff $\mathrm{K}$, Takeuchi A, Park IH, Sero JE, Zhu H, Perez-Atayde A, Frazier AL, et al. 2009. A role for Lin28 in primordial germ-cell development and germ-cell malignancy. Nature 460: 909-913.

Yang DH, Moss EG. 2003. Temporally regulated expression of Lin-28 in diverse tissues of the developing mouse. Gene Expr Patterns 3: 719-726.

Zhou J, Ng SB, Chng WJ. 2013. LIN28/LIN28B: an emerging oncogenic driver in cancer stem cells. Int J Biochem Cell Biol 45: 973-978.

Zhu H, Shyh-Chang N, Segre AV, Shinoda G, Shah SP, Einhorn WS, Takeuchi A, Engreitz JM, Hagan JP, Kharas MG, et al. 2011. The Lin28/let-7 axis regulates glucose metabolism. Cell 147: 81-94. 


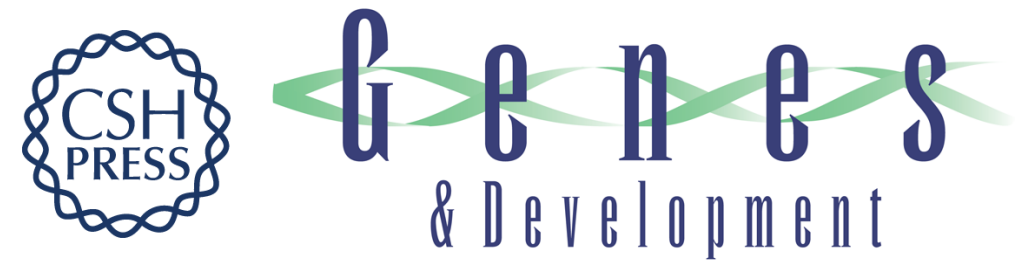

\section{LIN28 cooperates with WNT signaling to drive invasive intestinal and colorectal adenocarcinoma in mice and humans}

Ho-Chou Tu, Sarah Schwitalla, Zhirong Qian, et al.

Genes Dev. 2015, 29: originally published online May 8, 2015

Access the most recent version at doi:10.1101/gad.256693.114

\section{Supplemental http://genesdev.cshlp.org/content/suppl/2015/05/01/gad.256693.114.DC1 Material}

References This article cites 65 articles, 21 of which can be accessed free at: http://genesdev.cshlp.org/content/29/10/1074.full.html\#ref-list-1

Creative This article is distributed exclusively by Cold Spring Harbor Laboratory Press for the first Commons six months after the full-issue publication date (see

License http://genesdev.cshlp.org/site/misc/terms.xhtml). After six months, it is available under a Creative Commons License (Attribution-NonCommercial 4.0 International), as described at http://creativecommons.org/licenses/by-nc/4.0/.

Email Alerting Receive free email alerts when new articles cite this article - sign up in the box at the top Service right corner of the article or click here.

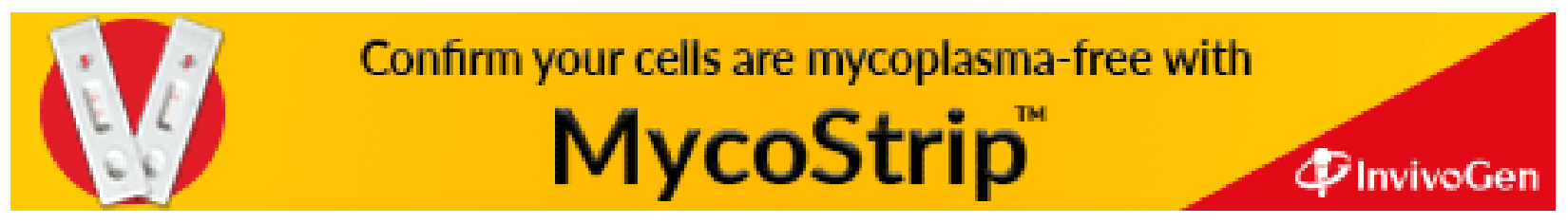

